Pacific Journal of Mathematics

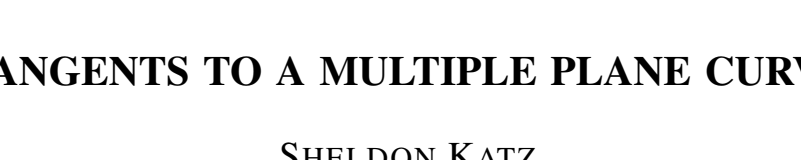




\title{
TANGENTS TO A MULTIPLE PLANE CURVE
}

\author{
SHELDON KaTZ
}

The limiting behavior of the tangents and the flexes are computed as a reduced plane curve degenerates into a multiple plane curve.

0. Introduction. In this paper, we consider the degeneration of a reduced irreducible plane curve to a multiple plane curve. We study the associated degeneration of tangent lines by viewing a line as a linear imbedding $\mathbf{P}^{1} \hookrightarrow \mathbf{P}^{2}$ and studying deformations of this imbedding. We compute the limiting behavior of the dual curve and the flexes. A similar computation yields the limiting behavior of the bitangents; this will appear later in a separate paper. The main result is stated as Proposition (2.1).

The author takes this opportunity to thank Professor William Fulton for introducing him to this problem.

1. The dual of a multiple curve. Let $C \subset \mathbf{P}_{\mathbf{C}}^{2}$ be a smooth curve of degree $d . C^{*} \subset \mathbf{P}^{2 *}$ will denote the dual curve of tangents to $C$.

Let $n$ be a positive integer, $n \geq 2$. Let

$$
G^{n}+t F=0
$$

be a generic pencil of plane curves, with $\operatorname{deg} G=d, \operatorname{deg} F=n d$. We will freely abuse notation by using the same letter to denote a polynomial or its zero locus. Here, generic means that $G, F$ are smooth, and meet transversely at their $n d^{2}$ points of intersection, the base points of the pencil. $G^{*}$ is assumed to have only nodes and cusps as singularities. The pencil (1.1) will be denoted by $C_{t}$. Let $C_{0}^{*}=\lim _{t \rightarrow 0} C_{t}^{*}$. The goal of this section is to prove the following.

(1.2) Proposition. $C_{0}^{*}$ is the union of $G^{*}$ with multiplicity $n$, together with the $n d^{2}$ pencils of lines through the base points, each pencil having multiplicity $(n-1)$.

Remarks. (1) Proposition (1.2) is quite elementary. It is not much more difficult than the case $n=2, d=1$ implicitly worked out in [4]. The 
value in this method of proof lies purely in its expository value as a prelude to $\$ 2$.

(2) By a standard formula for plane curves ([2], for example) $\operatorname{deg} C_{t}^{*}$ $=n d(n d-1)$ for $t \neq 0$, while $\operatorname{deg} C_{0}^{*}=n d(d-1)+(n-1) n d^{2}=$ nd $($ nd -1$)$.

The techniques used are a variant of the techniques of [3], which were inspired by the work of Clemens. Given a line $L \subset \mathbf{P}^{2}$, we look for a family of lines $L_{s}$ with $L_{0}=L$ and $L_{s}$ tangent to $C_{t}$ with $t=s^{r}$ for a positive integer $r$. Then $L$ would correspond to a general point of a multiplicity $r$ component of $C_{0}^{*}$ with cyclic local monodromy.

We choose an isomorphism $\alpha: \mathbf{P}^{1} \rightarrow L$ given by three homogeneous linear forms $\alpha=\left(\alpha_{0}(u, v), \alpha_{1}(u, v), \alpha_{2}(u, v)\right)$, where $(u, v)$ are homogeneous coordinates on $\mathbf{P}^{1}$. We single out $(1,0) \in \mathbf{P}^{1}$ as the candidate for a point of tangency of $L$ with $C_{0}$. We look for an extension of $\alpha$ to $\alpha(s)$, holomorphic in $s$ for $|s|<\varepsilon$, with $\alpha(0)=\alpha$, and satisfying

$$
\left(G^{n}+s^{r} F\right) \circ \alpha(s) \equiv 0\left(v^{2}\right) \text { for }|s|<\varepsilon .
$$

We attempt to solve (1.3) by power series in $s$. We show that this is possible when either $L$ is tangent to $G$ or when $L$ passes through a base point. In the former case, for general $L$, we must take $r=n$, while in the latter case, we take $r=n-1$. By consideration of degrees, i.e. Remark (2), no other components are present, proving Proposition (1.2).

We now fix some more notation. Let $P_{k}$ denote the vector space of homogeneous forms of degree $k$ on $\mathbf{P}^{1}$. There is a linear map

$$
\Phi_{G}: P_{1}^{3} \rightarrow P_{d}, \Phi_{G}\left(\sigma_{0}, \sigma_{1}, \sigma_{2}\right)=\sum_{j=0}^{2} \sigma_{j}\left(\frac{\partial G}{\partial X_{j}} \circ \alpha\right)
$$

and for each integer $k \geq 0$, the related map

$$
\Phi_{G}^{(k)}: P_{1}^{3} \stackrel{\Phi_{G}}{\rightarrow} P_{d} \rightarrow P_{d} /\left(v^{k+1}\right) .
$$

(1.6) LeMma. For any $L, \Phi_{G}^{(1)}$ is surjective (hence also $\Phi_{G}^{(0)}$ ).

Proof. Since $G$ is smooth, we may change coordinates so $\psi=$ $\partial G / \partial X_{0} \circ \alpha \not \equiv 0(\mathrm{v})$, so that $\psi$ is a unit in the graded ring $R_{1}=\oplus_{j} P_{j} /\left(v^{2}\right)$. Thus any $Q \in P_{d} /\left(v^{2}\right)$ can be divided by $\psi\left(\bmod v^{2}\right)$ to yield $\sigma \in P_{1}$; then $\Phi_{G}^{(1)}(\sigma, 0,0)=Q$. 
We introduce some more notation to facilitate higher order computations. Let

$$
\alpha^{(r)}=\left(\left.\frac{d^{r} \alpha_{i}}{d s^{r}}\right|_{s=0}\right)_{i=0,1,2}, \quad G_{i j} \alpha^{(r)} \alpha^{(s)}=\sum_{i, j}\left(\frac{\partial^{2} G}{\partial X_{i} \partial X_{j}}\right) \alpha_{i}^{(r)} \alpha_{j}^{(s)} .
$$

We also note that homogeneous polynomials of degree $j$ in $(u, v)$ can be viewed as polynomials of degree $\leq j$ in $v$; we will hence usually view $P_{j} /\left(v^{k+1}\right) \subset \mathrm{C}[v] /\left(v^{k+1}\right)$, and speak of constant terms, linear terms, etc. We also freely divide truncated polynomials.

We start by specializing to the case $n=2$ to fix ideas.

(1.7) Proposition. (1.2) is true for $n=2$.

Proof. We set $n=2, r=1$ (so that $s=t$ ) in (1.3), and let $t=0$ to obtain

$$
G^{2} \equiv 0\left(v^{2}\right)
$$

where we have abused notation by viewing $G$ as a form on $\mathbf{P}^{1}$ via $\alpha$. This gives

$$
G \equiv 0(v) \text {. }
$$

We continue by differentiating (1.3) with respect to $t$ and setting $t=0$.

$$
2 G \Phi_{G} \alpha^{\prime}+F \equiv 0\left(v^{2}\right)
$$

Using (1.9), (1.10) forces $F=0(v)$, i.e.

$$
L \text { passes through a base point. }
$$

To show that the pencil containing $L$ indeed has multiplicity 1 in $C_{0}^{*}$, we may take $L$ general, and so assume $G$ is not tangent to $L \simeq \mathbf{P}^{1}$ at $(1,0)$. We then obtain from (1.10)

$$
\Phi_{G}^{(0)} \alpha^{\prime}=-F / 2 G
$$

and Lemma 1.6 implies that we can solve (1.12) for $\alpha^{\prime}$. Thus the pencils through the base points deform to first order; these pencils are the only candidates for a multiplicity 1 component of $C_{0}^{*}$.

For the second order obstruction, we take the second derivative of (1.3) with respect to $t$ and set $t=0$ to obtain

$$
2 G \Phi_{G} \boldsymbol{\alpha}^{\prime \prime}+2 G G_{i j} \boldsymbol{\alpha}^{\prime} \boldsymbol{\alpha}^{\prime}+2\left(\Phi_{G} \boldsymbol{\alpha}^{\prime}\right)^{2}+2 \Phi_{F} \boldsymbol{\alpha}^{\prime} \equiv 0\left(v^{2}\right)
$$


In order for (1.13) to have a solution for $\alpha^{\prime \prime}$, we must require that

$$
2\left(\Phi_{G} \alpha^{\prime}\right)^{2}+2 \Phi_{F}\left(\alpha^{\prime}\right) \equiv 0(v) .
$$

This can be accomplished by the following lemma.

(1.15) LeMmA. $\left.\Phi_{F}^{(0)}\right|_{\operatorname{ker} \Phi_{G}^{(1)}}: \operatorname{ker} \Phi_{G}^{(1)} \rightarrow P_{n d} /(v)$ is surjective.

Proof. Since $\operatorname{dim} P_{n d} /(v)=1$, the lemma can fail to hold only if $\operatorname{ker} \Phi_{G}^{(1)} \subset \operatorname{ker} \Phi_{F}^{(0)}$. But since $F$ and $G$ intersect transversally, we can change coordinates in $\mathbf{P}^{2}$ so that $X_{0}=0$ is tangent to $F$, and $X_{1}=0$ is tangent to $G$ at $\alpha(1,0)$. So we may assume that, in the affine coordinate $v$ near $(1,0) \in \mathbf{P}^{1}, \quad\left(\partial G / \partial X_{0}\right)(\alpha(v)) \equiv a v\left(v^{2}\right), \quad\left(\partial G / \partial X_{1}\right)(\alpha(v)) \equiv b(v)$, where $b \neq 0$. Then $(-b u, a v, 0) \in \operatorname{ker} \Phi_{G}^{(1)}-\operatorname{ker} \Phi_{F}^{(0)}$.

Now we can replace $\alpha^{\prime}$ with $\alpha^{\prime}-\tilde{\alpha}$, where $\tilde{\alpha} \in \operatorname{ker} \Phi_{G}^{(1)}$ and $\Phi_{F}^{(0)} \tilde{\alpha} \equiv$ $\left(\Phi_{G} \alpha^{\prime}\right)^{2}(v)$, by the lemma. Then (1.12) still holds, but now the left-hand side of (1.13) is divisible by $G$, since (1.14) now holds. After dividing (1.13) by $G$, we can now solve for $\alpha^{\prime \prime}$ by using lemma (1.6) again.

For simplicity, we introduce the symbol $Q_{j}$ to stand for any expression involving $\alpha$ only through $\alpha^{\prime}, \alpha^{\prime \prime}, \ldots, \alpha^{(j)}$. The higher order obstructions are now handled by the following easily established lemma.

(1.16) LemMA. For $n \geq 2$, the nth obstruction to (1.3) is

$$
\begin{aligned}
\left.\frac{d^{n}}{d t^{n}}\left(G^{2}+t F\right)\right|_{t=0} \equiv & 2 G \Phi_{G} \alpha^{(n)}+n \Phi_{F} \alpha^{(n-1)} \\
& +2 n \Phi_{G} \alpha^{\prime} \Phi_{G} \alpha^{(n-1)}+G Q_{n-1}+Q_{n-2} \equiv 0\left(v^{2}\right) .
\end{aligned}
$$

We inductively complete the power series solution of (1.3). We suppose that we have solved for $\alpha^{\prime}, \ldots, \alpha^{(n-1)}$. Then using Lemma 1.15, we modify $\alpha^{(n-1)}$ so that $(1.16)$ becomes divisible by $G$. After dividing by $G$, we use Lemma (1.6) once more to solve for $\alpha^{(n)}$.

This procedure gives a formal power series solution of (1.3). By Artin's theorem [1] there is a holomorphic solution of (1.3) for $|t|<\varepsilon$. Thus, the pencils through the base points are each multiplicity 1 components of $C_{0}^{*}$.

REMARK. The solution for $\alpha^{(n)}$ is far from unique; in fact, the computation above shows that the ambiguity lies in $\operatorname{ker} \Phi_{G}^{(0)} \cap \operatorname{ker} \Phi_{F}^{(0)}$, a 4-dimensional vector space. Let $B \subset \mathrm{GL}(2)$ denote the isotropy group of 
$(1,0)$, so that $\operatorname{dim} B=3$. This is the ambiguity arising by representing $L$ as $\left(\mathbf{P}^{1},(1,0)\right)$. The difference between 4 and 3 reflects that a curve (the pencil) is deforming.

The other component $2 G^{*}$ is found by letting $n=2, t=s^{2}$ in (1.3). The order zero obstruction again leads to (1.9), which holds for a tangent to $G$ (in fact, $G \equiv 0\left(v^{2}\right)$ ). The first order obstruction is

$$
2 G \Phi_{G} \alpha^{\prime} \equiv 0\left(v^{2}\right)
$$

which is again automatic, and puts no restrictions on $\alpha^{\prime}$.

The second order obstruction is

$$
2 G \Phi_{G} \alpha^{\prime \prime}+2\left(\Phi_{G} \alpha^{\prime}\right)^{2}+2 G G_{i j} \alpha^{\prime} \alpha^{\prime}+2 F \equiv 0\left(v^{2}\right) .
$$

This equation can be solved for $\alpha^{\prime \prime}$ provided that

$$
\left(\Phi_{G} \alpha^{\prime}\right)^{2} \equiv-F\left(v^{2}\right) .
$$

We can assume that $L$ does not pass through a base point (i.e. $F \not \equiv 0(v)$ ). After taking a square root, Lemma (1.6) ensures that we can find such an $\alpha^{\prime}$, and (1.18) imposes no conditions on $\alpha^{\prime \prime}$. For the higher order obstructions we need an easy lemma.

(1.20) LEMMA. For $n \geq 2$, the nth obstruction is

$$
\begin{aligned}
\left.\frac{d^{n}}{d s^{n}}\left(G^{2}+s^{2} F\right)\right|_{s=0} \equiv & 2 G \Phi_{G} \alpha^{(n)}+2 n \Phi_{G} \alpha^{\prime} \Phi_{G} \alpha^{(n-1)} \\
& +G Q_{n-1}+Q_{n-2} \equiv 0\left(v^{2}\right) .
\end{aligned}
$$

Since $\Phi_{G} \alpha^{\prime}$ is a unit in $P_{d} /\left(v^{2}\right)$, we can choose $\alpha^{(n-1)}$ to ensure that there is no $n$th obstruction, using Lemma (1.6). Thus, there is a formal power series solution of (1.3) with $t=s^{2}$, and Artin's Theorem finishes the proof of Proposition (1.7).

REMARK. In the case of tangents, the ambiguity lies in $\operatorname{ker} \Phi_{G}^{(1)}$, which is as before a 4-dimensional vector space.

Proof of Proposition (1.2). We start by letting $t=s^{n-1}$ in (1.3), and attempt to deform a pencil through a base point. There are clearly no obstructions through order $n-2$. The $(n-1)$ st obstruction is (since 
$G^{j} \equiv 0\left(v^{2}\right)$ for $\left.j \geq 2\right)$

$$
n ! G\left(\Phi_{G} \alpha^{\prime}\right)^{n-1}+(n-1) ! F \equiv 0\left(v^{2}\right) .
$$

We may assume $L$ is not tangent to $G$ or $F$; then we can solve (1.21) for $\boldsymbol{\alpha}^{\prime}$.

The $n$th order obstruction is seen to be

$$
\begin{gathered}
n !\left(\begin{array}{l}
n \\
2
\end{array}\right) G\left(\Phi_{G} \alpha^{\prime}\right)^{n-2} \Phi_{G} \alpha^{\prime \prime}+n !\left(\Phi_{G} \alpha^{\prime}\right)^{n}+n ! \Phi_{F} \alpha^{\prime} \\
+G Q_{n} \equiv 0\left(v^{2}\right) .
\end{gathered}
$$

We now can use Lemma (1.15) to modify $\alpha^{\prime}$ so that (1.22) is consistent. After dividing (1.22) by $G$, and noting that $\Phi_{G}^{(1)} \alpha^{\prime}$ is a unit, we can then solve for $\alpha^{\prime \prime}$.

For the higher order obstructions, we note that for $r \geq n+1$

$$
\begin{aligned}
& \left.\frac{d^{r}}{d s^{r}}\left(G^{n}+s^{n-1} F\right)\right|_{s=0} \\
& \equiv n(n-1) \frac{r !}{(r-n+2) !} G\left(\Phi_{G} \alpha^{\prime}\right)^{n-2} \Phi_{G} \alpha^{(r-n+2)} \\
& \quad+n \frac{r !}{(r-n+2) !}\left(\Phi_{G} \alpha^{\prime}\right)^{n-1} \Phi_{G} \alpha^{(r-n+1)} \\
& \quad+\frac{r !}{(r-n+1) !} \Phi_{F} \alpha^{(r-n+1)}+G Q_{r-n+1}+Q_{r-n} \equiv 0\left(v^{2}\right) .
\end{aligned}
$$

As before, we can use Lemma (1.15) inductively to modify $\alpha^{(r-n+1)}$ to ensure the consistency of (1.23), then solve for $\alpha^{(r-n+2)}$ using Lemma (1.6). Finally, Artin's Theorem shows that a pencil through a base point is a multiplicity $(n-1)$ component of $C_{0}^{*}$.

Turning next to the tangents to $G$ (so that $G \equiv 0\left(v^{2}\right)$ ), we let $t=s^{n}$ in (1.3). There are clearly no obstructions through order $(n-1)$.

The $n$th order obstruction yields

$$
n !\left(\Phi_{G} \alpha^{\prime}\right)^{n}+n ! F \equiv 0\left(v^{2}\right) .
$$

Assuming that $L$ does not pass through a base point, we can solve (1.24) for $\alpha^{\prime}$.

For the higher order obstructions, we note that for $r \geq n+1$

$$
\begin{aligned}
\left.\frac{d^{r}}{d s^{r}}\left(G^{n}+s^{n} F\right)\right|_{s=0} \equiv & n \frac{r !}{(r-n+1) !}\left(\Phi_{G} \alpha^{\prime}\right)^{n-1} \Phi_{G} \alpha^{(r-n+1)} \\
& +Q_{r-n} \equiv 0\left(v^{2}\right)
\end{aligned}
$$


As $\Phi_{G} \alpha^{\prime}$ is a unit, we can solve for $\alpha^{(r-n+1)}$. Artin's Theorem completes the proof.

2. Flexes on a multiple curve. In the situation of $\S 1$, we look at the limiting behavior of the flexes of $C_{t}$.

(2.1) Proposition. The flexes of $C_{t}$ degenerate to the flexes of $G$, the tangents to $F$ at a base point, and the tangents to $G$ at a base point, with multiplicities $n, n-2,2 n-1$ respectively.

Proof. By a standard formula for plane curves [2], $C_{t}$ has $3 n d(n d-2)$ flexes; $g$ has $3 d(d-2)$ flexes and $n d^{2}$ base points. Also $3 n d(n d-2)=$ $n(3 d(d-2))+(n-2) n d^{2}+(2 n-1) n d^{2}$. So as in $\S 1$, it suffices to construct deformations of the claimed limits with the indicated multiplicities.

We now need to solve

$$
\left(G^{n}+s^{r} F\right) \circ \alpha(s) \equiv 0\left(v^{3}\right) \text { for }|s|<\varepsilon
$$

for $r=n$ in the case of a flex of $G$, for $r=n-2$ in the case of a tangent to $F$ at a base point, and for $r=2 n-1$ in the case of a tangent to $G$ at a base point.

We first check the flexes of $G$, starting with a lemma.

(2.3) LeMMA. If $L$ is an ordinary inflectional tangent to $G$, then $\Phi_{G}^{(2)}$ is surjective.

Proof. We can change coordinates so that $L$ has equation $X_{1}=0$, and $G$ has an equation of the form $X_{1} f+X_{0}^{3} g$, where $f(0,0,1), g(0,0,1)$ $\neq 0$. We may as well let $\alpha: \mathbf{P}^{1} \rightarrow L$ be $\alpha(u, v)=(v, 0, u)$. Then, using subscript notation for partial derivatives, we find that

$$
G_{0} \circ \alpha=3 v^{2} g+v^{3} g_{0} \quad G_{1} \circ \alpha=f+v^{3} g_{1}
$$

and so $\Phi_{G}^{(2)}$ is surjective by inspection.

The proof of the case of flexes is now completed by mimicking the computation of the component $n G^{*}$ of $\S 1$, using Lemma (2.3) in place of Lemma (1.6).

We turn next to the case of a tangent to $F$ at a base point, i.e. $G \equiv 0$ $(v), F \equiv 0\left(v^{2}\right), t=s^{n-2}$. 
There are clearly no obstructions through order $n-3$. For the order $n-2$ obstruction, we note that

$$
\left.\frac{d^{n-2}}{d s^{n-2}}\left(G^{n}+s^{n-2} F\right)\right|_{s=0} \equiv \frac{n !}{2} G^{2}\left(\Phi_{G} \alpha^{\prime}\right)^{n-2}+(n-2) ! F \equiv 0\left(v^{3}\right)
$$

and since $F, G$ have order exactly 2,1 respectively as polynomials in $v$, $F / G^{2}$ is a unit, so we can extract an $(n-2)$ root and solve for $\Phi_{G}^{(0)} \alpha^{\prime}$ in (2.4).

The higher order obstructions are given by

$$
\begin{aligned}
& \left.\frac{d^{k}}{d s^{k} k}\left(G^{n}+s^{n-2} F\right)\right|_{s=0} \\
& =\frac{n(n-1)(n-2)}{2} \frac{k !}{(k+3-n) !} G^{2}\left(\Phi_{G} \alpha^{\prime}\right)^{n-3} \Phi_{G} \alpha^{(k+3-n)} \\
& \quad+G^{2} Q_{k+2-n}+n(n-1) \frac{k !}{(k+2-n) !} G\left(\Phi_{G} \alpha^{\prime}\right)^{n-2} \Phi_{G} \alpha^{(k+2-n)} \\
& \quad+\frac{k !}{(k+2-n) !} \Phi_{F} \alpha^{(k+2-n)}+Q_{k+1-n}\left(v^{3}\right)
\end{aligned}
$$

This case is finished by a couple of lemmas.

(2.6) LeMMA. $\left.\Phi_{F}^{(1)}\right|_{\operatorname{ker} \Phi_{G}^{(0)}}: \operatorname{ker} \Phi_{G}^{(0)} \rightarrow P_{n d} /\left(v^{2}\right)$ is surjective.

Proof. Lemma 1.15 says that $\operatorname{dim} \operatorname{ker} \Phi_{G}^{(1)} \cap \operatorname{ker} \Phi_{F}^{(0)}=3$. Reversing the roles of $F$ and $G$ yields the lemma.

(2.7) LemMa. After solving for the kth obstruction, we have $\infty^{3}$ solutions for $\boldsymbol{\alpha}^{1}, \ldots, \boldsymbol{\alpha}^{(k+2-n)}$, and $\Phi_{G}^{(0)} \boldsymbol{\alpha}^{(k+3-n)}$ is determined.

Proof. Inductively, we equate the linear plus constant term of (2.5) to $0\left(v^{2}\right)$, using Lemma (2.6) to modify $\alpha^{(k+2-n)} . \Phi_{G}^{(0)} \alpha^{(k+3-n)}$ is now found by Lemma (1.6).

An application of Artin's Theorem completes the proof of the case of a tangent to $F$ at a base point.

Finally, we turn to a tangent to $G$ at a base point, i.e. $G \equiv 0\left(v^{2}\right)$, $F \equiv 0(v), t=s^{2 n-1}$.

There are clearly no obstructions through order $n-2$. The order $n-1$ obstruction is

$$
\left.\frac{d^{n-1}}{d s^{n-1}}\left(G^{n}+s^{2 n-1} F\right)\right|_{s=0} \equiv n ! G\left(\Phi_{G} \alpha^{\prime}\right)^{n-1} \equiv 0\left(v^{3}\right)
$$


which forces

$$
\Phi_{G}^{(0)} \alpha^{\prime}=0 .
$$

We change notation slightly, putting $G^{(j)}=d^{j}(G \circ \alpha(s)) /\left.d s^{j}\right|_{s=0}$, noting that $G^{(j)}=\Phi_{G} \alpha^{(j)}+Q_{j-1}$. With the additional information (2.9), we now see that there are no obstructions through order $2 n-3$. The order $2 n-2$ obstruction is given by

$$
\begin{aligned}
& \left.\frac{d^{2 n-2}}{d s^{2 n-2}}\left(G^{n}+s^{2 n-1} F\right)\right|_{s=0} \\
& \quad=\frac{n(2 n-2) !}{2^{n-1}} G\left(G^{\prime \prime}\right)^{n-1}+\frac{n(n-1)(2 n-2) !}{2^{n-1}}\left(G^{\prime}\right)^{2}\left(G^{\prime \prime}\right)^{n-2} \\
& \quad \equiv 0\left(v^{3}\right) .
\end{aligned}
$$

This leads to

$$
G^{\prime \prime} \equiv-(n-1)\left(G^{\prime}\right)^{2} / G(v) .
$$

The order $2 n-1$ obstruction is

$$
\begin{aligned}
\frac{d^{2 n-1}}{d s^{2 n-1}} & \left.\left(G^{n}+s^{2 n-1} F\right)\right|_{s=0} \\
= & \frac{n(n-1)(2 n-1) !}{6 \cdot 2^{n-2}} G\left(G^{\prime \prime}\right)^{n-2} G^{\prime \prime \prime} \\
& +\frac{n(n-1)(n-2)(2 n-1) !}{6 \cdot 2^{n-2}}\left(G^{\prime}\right)^{2}\left(G^{\prime \prime}\right)^{n-3} G^{\prime \prime \prime} \\
& +\frac{n(2 n-1) !}{2^{n-1}} G^{\prime}\left(G^{\prime \prime}\right)^{n-1}+(2 n-1) ! F \\
\equiv & 0\left(v^{3}\right)
\end{aligned}
$$

looking at the linear term, and using (2.11), we find

$$
\left(G^{\prime}\right)^{2 n-1} / G^{n-1} \equiv(-1)^{n} \frac{2^{n-1}}{n(n-1)^{n-1}} F\left(v^{2}\right) .
$$

(2.12) implies that we can solve for $\Phi_{G}^{(1)} \alpha^{\prime}$, and that $G^{\prime \prime}$ is a unit, using (2.11) again.

Turning to the quadratic term of (2.12), we see that we must solve for $G^{\prime \prime \prime}(v)$, or equivalently, for $\Phi_{G}^{(0)} \boldsymbol{\alpha}^{\prime \prime \prime}$. This is possible exactly when the expression multiplying $G^{\prime \prime \prime}$ in $(2.12)$ is divisible by $v^{2}$, but not by $v^{3}$. But this expression is a multiple of

$$
\left(G^{\prime \prime}\right)^{n-3}\left[G G^{\prime \prime}+(n-2)\left(G^{\prime}\right)^{2}\right]
$$


which satisfies the indicated requirement, by (2.11) and the fact that $G^{\prime \prime}$ is a unit.

Notice that $\Phi_{G}^{(0)} \alpha^{\prime \prime}$ depends only on $\alpha^{\prime}$, while $\Phi_{G}^{(0)} \alpha^{\prime \prime \prime}$ depends on $\Phi_{G}^{(1)} \alpha^{\prime \prime}$ and $\alpha^{\prime}$; however, it is a non-trivial linear expression in the linear term of $\Phi_{G}^{(1)} \alpha^{\prime \prime}$, as revealed by a examination of our solution of (2.12).

The higher order obstructions are given by

$$
\begin{aligned}
& \left.\frac{d^{k}}{d s^{k}}\left(G^{n}+s^{2 n-1} F\right)\right|_{s=0} \\
& =\frac{n(n-1) k !}{2^{n-2}(k+4-2 n) !} G\left(G^{\prime \prime}\right)^{n-2} G^{(k+4-2 n)} \\
& \quad+\frac{n(n-1)(n-2) k !}{2^{n-2}(k+4-2 n) !}\left(G^{\prime}\right)^{2}\left(G^{\prime \prime}\right)^{n-3} G^{(k+4-2 n)}+G Q_{k+3-2 n} \\
& \quad+\left(G^{\prime}\right)^{2} \tilde{Q}_{k+3-2 n}+\frac{n(n-1) k !}{2^{n-2}(k+3-2 n) !} G^{\prime}\left(G^{\prime \prime}\right)^{n-2} G^{(k+3-2 n)} \\
& \quad+G^{\prime} Q_{k+2-2 n}+\frac{n k !}{2^{n-1}(k+2-2 n) !}\left(G^{\prime \prime}\right)^{n-1} G^{(k+2-2 n)} \\
& \quad+Q_{k+1-2 n}+\frac{k !}{(k+1-2 n) !} \Phi_{F}\left(\alpha^{(k+1-2 n)}\right) \\
& \equiv 0\left(v^{3}\right) .
\end{aligned}
$$

Equation (2.15) can be solved inductively.

(2.16) LEMma. After solving for the kth obstruction, we have $\infty^{3}$ solutions for $\alpha^{\prime}, \ldots, \alpha^{(k+1-2 n)}$, we have found $\Phi_{G}^{(1)} \alpha^{(k+2-2 n)}$, and we have found $\Phi_{G}^{(0)} \alpha^{(k+4-2 n)}$. This last depends non-trivially and linearly on the linear term of $\Phi_{G} \alpha^{(k+3-2 n)}$, and on terms of lower order.

Proof. By induction. We start by examining the constant term of (2.15). We observe that the constant term of $G^{(k+2-2 n)}$ depends on $\boldsymbol{\Phi}_{G}^{(1)} \boldsymbol{\alpha}^{(k+1-2 n)}$ and lower derivatives of $\boldsymbol{\alpha}$. Also we note that the expression $Q_{\mathrm{k}+1-2 n}$ in (2.15) depends on $\Phi_{G}^{(0)} \alpha^{(k+1-2 n)}$ and lower derivatives of $\alpha$. So Lemma (1.15) applies to allow for the modification of $\alpha^{(k+1-2 n)}$ as before.

Next, we consider the linear term of (2.15). We observe that the constant term of $Q_{\mathrm{k}+2-2 n}$ depends on $\Phi_{G}^{(0)} \alpha^{(k+2-2 n)}$ and lower derivatives of $\alpha$, while inductively the constant term of $G^{(k+3-2 n)}$ depends non-trivially and linearly on the linear term of $\Phi_{G} \alpha^{(k+2-2 n)}$ and on lower order 
terms, so that after equating the linear term of (2.15) to 0 , we can first solve for the linear term of $\Phi_{G} \alpha^{(k+2-2 n)}$ (hence for $\Phi_{G}^{(1)} \alpha^{(k+2-2 n)}$, as we inductively know the constant term). Lemma (1.16) allows us to solve for $\alpha^{(k+2-2 n)}$.

Finally, we turn to the quadratic term. Exactly as in the order $2 n-1$ obstruction, we see that $\Phi_{G}^{(0)} \alpha^{(k+4-2 n)}$ is multiplied by a constant multiple of (2.14), which we have seen is divisible by $v^{2}$, but not by $v^{3}$. So we can solve for $\Phi_{G}^{(0)} \alpha^{(k+4-2 n)}$, and apply Lemma (1.6). Note that the quadratic term of (2.15) involves $\alpha^{(k+3-2 n)}$ only non-trivially and linearly through the linear term of $\Phi_{G} \alpha^{(k+3-2 n)}$, completing the induction.

An application of Artin's Theorem now finishes the case of tangents to $G$ through a base point, as well as the proof of Proposition (2.1).

\section{REFERENCES}

[1] M. Artin, On the solution of analytic equations, Invent. Math., 5 (1968), 277-291.

[2] P. Griffiths and J. Harris, Principles of Algebraic Geometry, Wiley-Interscience, N.Y. 1978.

[3] S. Katz, Degeneration of quintic threefolds and their lines, Duke Math. J., 50 (1983), 1127-1135.

[4] H. Schubert, Kalkül der Abzählenden Geometrie, Springer-Verlag, Berlin, 1979.

Received April 25, 1985.

UNIVERSITY OF OKLAHOMA

NORMAN, OK 73019 



\section{PACIFIC JOURNAL OF MATHEMATICS EDITORS}

\author{
V. S. VARADARAJAN \\ (Managing Editor) \\ University of California \\ Los Angeles, CA 90024 \\ Herbert Clemens \\ University of Utah \\ Salt Lake City, UT 84112 \\ R. FINN \\ Stanford University \\ Stanford, CA 94305
}

\author{
HERMANN FLASCHKA \\ University of Arizona \\ Tucson, AZ 85721 \\ RAMESH A. GANGOLLI \\ University of Washington \\ Seattle, WA 98195 \\ VAUGHAN F. R. JONES \\ University of California \\ Berkeley, CA 94720 \\ ROBION KIRBY \\ University of California \\ Berkeley, CA 94720
}

C. C. MOORE

University of California

Berkeley, CA 94720

H. SAMELSON

Stanford University

Stanford, CA 94305

HAROLD STARK

University of California, San Diego La Jolla, CA 92093

\section{ASSOCIATE EDITORS}
R. ARENS
E. F. BECKENBACH (1906-1982)
B. H. NEUMANN
F. WOLF
K. YosHIDA

\section{SUPPORTING INSTITUTIONS}

\begin{abstract}
UNIVERSITY OF ARIZONA
UNIVERSITY OF BRITISH COLUMBIA

CALIFORNIA INSTITUTE OF TECHNOLOGY

UNIVERSITY OF CALIFORNIA

MONTANA STATE UNIVERSITY

UNIVERSITY OF NEVADA, RENO

NEW MEXICO STATE UNIVERSITY OREGON STATE UNIVERSITY
\end{abstract}

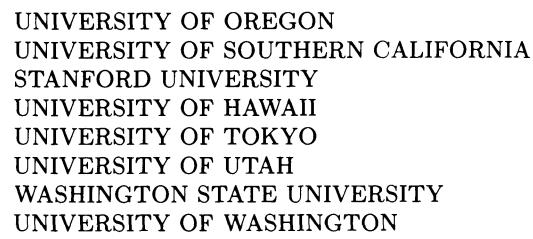

The Supporting Institutions listed above contribute to the cost of publication of this Journal, but they are not owners or publishers and have no responsibility for its content or policies.

Mathematical papers intended for publication in the Pacific Journal of Mathematics should be in typed form or offset-reproduced (not dittoed), double spaced with large margins. Please do not use built up fractions in the text of the manuscript. However, you may use them in the displayed equations. Underline Greek letters in red, German in green, and script in blue. The first paragraph must be capable of being used separately as a synopsis of the entire paper. In particular it should contain no bibliographic references. Please propose a heading for the odd numbered pages of less than 35 characters. Manuscripts, in triplicate, may be sent to any one of the editors. Please classify according to the scheme of Math. Reviews, Index to Vol. 39. Supply name and address of author to whom proofs should be sent. All other communications should be addressed to the managing editor, or Elaine Barth, University of California, Los Angeles, California 90024.

There are page-charges associated with articles appearing in the Pacific Journal of Mathematics. These charges are expected to be paid by the author's University, Government Agency or Company. If the author or authors do not have access to such Institutional support these charges are waived. Single authors will receive 50 free reprints; joint authors will receive a total of 100 free reprints. Additional copies may be obtained at cost in multiples of 50 .

The Pacific Journal of Mathematics is issued monthly as of January 1966. Regular subscription rate: $\$ 190.00$ a year (5 Vols., 10 issues). Special rate: $\$ 95.00$ a year to individual members of supporting institutions.

Subscriptions, orders for numbers issued in the last three calendar years, and changes of address should be sent to Pacific Journal of Mathematics, P.O. Box 969, Carmel Valley, CA 93924, U.S.A. Old back numbers obtainable from Kraus Periodicals Co., Route 100, Millwood, NY 10546.

The Pacific Journal of Mathematics at P.O. Box 969, Carmel Valley, CA 93924 (ISSN 0030-8730) publishes 5 volumes per year. Application to mail at Second-class postage rates is pending at Carmel Valley, California, and additional mailing offices. Postmaster: send address changes to Pacific Journal of Mathematics, P.O. Box 969, Carmel Valley, CA 93924.

PUBLISHED BY PACIFIC JOURNAL OF MATHEMATICS, A NON-PROFIT CORPORATION Copyright (C) 1986 by Pacific Journal of Mathematics 


\section{Pacific Journal of Mathematics}

Vol. 124, No. $2 \quad$ June, 1986

Philip Lee Bowers, Nonshrinkable "cell-like" decompositions of $s \ldots \ldots .257$

Aurelio Carboni and Ross Street, Order ideals in categories .......... 275

Leoni Dalla, Increasing paths on the one-skeleton of a convex compact set in

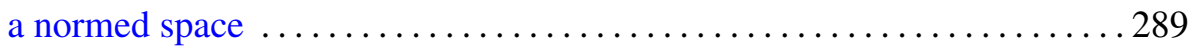

Jim Hoste, A polynomial invariant of knots and links ............... 295

Sheldon Katz, Tangents to a multiple plane curve ................... 321

Thomas George Lucas, Some results on Prüfer rings $\ldots \ldots \ldots \ldots \ldots \ldots 33$

Pham Anh Minh, Modular invariant theory and cohomology algebras of

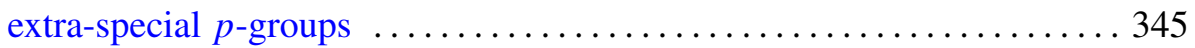

Ikuko Miyamoto, On inclusion relations for absolute Nörlund

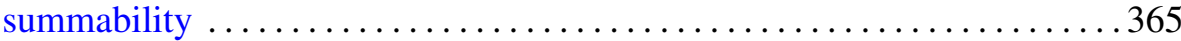

A. Papadopoulos, Geometric intersection functions and Hamiltonian flows on the space of measured foliations on a surface ............. 375

Richard Dean Resco, J. Toby Stafford and Robert Breckenridge

Warfield, Jr., Fully bounded $G$-rings $\ldots \ldots \ldots \ldots \ldots \ldots \ldots \ldots \ldots 4$

Haskell Paul Rosenthal, Functional Hilbertian sums .................417

Luen-Fai Tam, Regularity of capillary surfaces over domains with corners: borderline case ................................. 469

Hugh C. Williams, The spacing of the minima in certain cubic lattices .....483 